\title{
UNDERSTANDING DROPLET COALESCENCE AND ITS USE TO MEASURE INTERFACIAL TENSION
}

\author{
Claude VERDiER (ग), Matthieu BRIZARD \\ Laboratoire de Rhéologie* \\ BP 53 - Domaine Universitaire \\ F - 38041 GRENOBLE Cedex 9 \\ France \\ Verdier@ujf-grenoble.fr
}

Fax: (33) 476825164

Tel: (33) 476825294

\begin{abstract}
:
The study of coalescence of polymer droplets is presented, in the viscosity ratio range (p) going from 0.1 to 10 . It is shown that the determination of the characteristic time of collision is a good way to have access to the interfacial tension. Polydimethylsiloxane (PDMS) is mixed with Polyisobutylene (PIB) and the temperature change provides a way to modify the interfacial tension of the PDMS/PIB system significantly, as measured using a pendant drop apparatus. We obtain a dependence of the reduced collision time as a function of $\mathrm{p}^{-1 / 2}$ which gives access to the interfacial tension. This technique can be an interesting choice for measuring interfacial tension without requiring sophisticated techniques.

In a further attempt to correlate these observations with a theoretical model [Verdier C., Polymer, 42, 2001], the flow field inside and outside the droplets is investigated. PIV measurements are carried out where the evidence of elongational regimes is demonstrated. Such experiments are also interesting for future comparisons with numerical results.
\end{abstract}

Keywords : collision, polymer, elongation, viscosity ratio, PIV

\footnotetext{
* Université Joseph-Fourier, Institut National Polytechnique, CNRS (UMR 5520)
} 


\section{Introduction}

Understanding the mixing of two polymers requires to know about the rheological properties of the fluids, to be able to determine flow fields, but also to control the interfacial properties of such fluids. As a first start, one may study two newtonian fluids and look at droplet break-up and coalescence, since these two mechanisms govern the final properties of the mixture. Breakup has been studied by Taylor (1934) in his seminal paper and he found a criterion for breakup depending on the viscosity ratio of the fluids. Let us denote by $p$ the ratio $\eta_{1} / \eta_{2}$, with $\eta_{1}$ being the viscosity of the droplets (suspending fluid) and $\eta_{2}$ the viscosity of the matrix. At a

critical capillary number $\mathrm{Ca}=\frac{\eta_{2} \gamma \mathrm{Rf}(\mathrm{p})}{\sigma}$, the droplet undergoes high deformations, is elongated in the shear or elongational flow and eventually breaks into two parts. This result has been later improved experimentally by Grace (1982) who showed the variation of the critical capillary number $\mathrm{Ca}_{\mathrm{c}}$ as a function of $\mathrm{p}$ over ten decades. $\mathrm{f}(\mathrm{p})$ is an exact function of $\mathrm{p}$ and is equal to $(19 p+16) /(16 p+16)$, usually close to one (Taylor, 1934), $\mathrm{R}$ is the radius of the droplet and $\gamma$ is the shear rate. Also it was noticed that no breakup occurs in shear flows when $p$ is larger that 3.5 approximately. Finally, let us notice that when the shear rate is varied, one may expect a dependence of the final radius of the droplets as the inverse of the shear rate, since the critical

condition occurs at a fixed capillary number. In elongational flow situations, the shear rate $\gamma$ in

the capillary number is to be replaced with $\varepsilon$, which is the elongational rate, and a similar stability curve (Grace, 1982) is obtained with two separate regions corresponding to stable and unstable droplet.

Regarding the coalescence phenomenon, which is directly in competition with break-up during mixing, much less is known. Janssen and Meijer (1995) provide a similar criterion for the final droplet size during shearing and they give a relationship between the radius of the resulting droplets and the shear rate. A power dependence -0.6 is obtained. Comparing these behaviors in a shear flow (Grizzuti et al., 1997, Vinckier et al., 1998) can give information about which type of mechanisms (coalescence or break up or both) takes place when mixing two fluids.

Coalescence can also be regarded as an interesting mechanism, driven by interfacial tension, which can exist even when no shear is applied. Droplets can coalesce simply by interacting with each other. Usually, with polymers, van der Waals interactions are sufficient for droplets to merge and give a larger resulting droplet. Chesters (1991) studied this phenomenon in detail, in particular the approach between the droplets which gives rise to drainage of the interstitial fluid (fluid 2) until the droplets coalesce. Recently, Verdier $(2000,2001)$ paid attention to the flow involved by the coalescing droplets, after they come into contact, and studied the shape of the resulting droplet experimentally to determine the dependence of the collision time as a function of the viscosity ratio $\mathrm{p}$. Three regimes were found, each one with a typical slope as a function of $\mathrm{p}$. The most interesting one is the one obtained when the fluids have comparable viscosities $(0.1<\mathrm{p}<10)$, because such situations are commonly met in mixing devices. In this case the collision time varies like the geometric mean of the viscosities (Verdier, 2000).

This method is investigated here further in this paper, its aim being to find a new way to measure interfacial tension. This part is presented next, using two polymers (PDMS and PIB). Changing the temperature is a way to monitor the interfacial tension as well as viscosities. It is shown that the knowledge of the viscosities of the fluids and the collision time are sufficient to have access to the interfacial tension. The influence of the radius ratio between the droplets is also taken into account. This technique is checked with separate measurements of the interfacial 
tension using a pendant drop apparatus. The results are presented in the first section. In the second part of the experimental section, PIV measurements will also be shown: these measurements are indeed very important to investigate the flow field further, and may also be useful to compare with computations. It is shown that elongational flows are dominant close to center of the resulting droplet during collision. This method can finally be used further to calculate the viscous dissipation exactly during collision. It may be quite useful to determine the exact dependence of the collision time on $\mathrm{p}$ theoretically.

\section{Materials and experiments}

\section{Fluids and properties}

The two fluids which were selected are polydimethylsiloxane (PDMS, 47VT60000, RhônePoulenc, $\mathrm{Mw}=116,500 \mathrm{~g} / \mathrm{mole}, \mathrm{Mn}=62,400 \mathrm{~g} / \mathrm{mole}$ ) and polyisobutylene (PIB, Parapol 950, BP Chimie, $M w=1472 \mathrm{~g} / \mathrm{mole}, \mathrm{Mn}=932 \mathrm{~g} / \mathrm{mole})$. These types of fluids have been used previously (Grizzuti et al., 1997, Verdier, 2000, 2001) and provide adequate properties for visualization. Indeed their refraction indexes are close and allow visualization of one fluid inside the other and vice versa. Their rheological properties are known accurately and they are shown to behave like

newtonian fluids when the velocity gradient is small (typically $\gamma<1000 \mathrm{~s}^{-1}$ at $20^{\circ} \mathrm{C}$ ), which will be the case since times involved in the coalescence experiments are rather long (of the order of seconds). Experiments will be carried out at different temperatures ranging from $20^{\circ} \mathrm{C}$ to $60^{\circ} \mathrm{C}$, allowing for a viscosity ratio change, as well as changes in the interfacial tension. The viscosities are indeed required for the investigation of the collision time (Verdier, 2000). Figure 1 shows this dependence of the viscosity as a function of temperature.

\section{$\eta$ (Pa.s)}

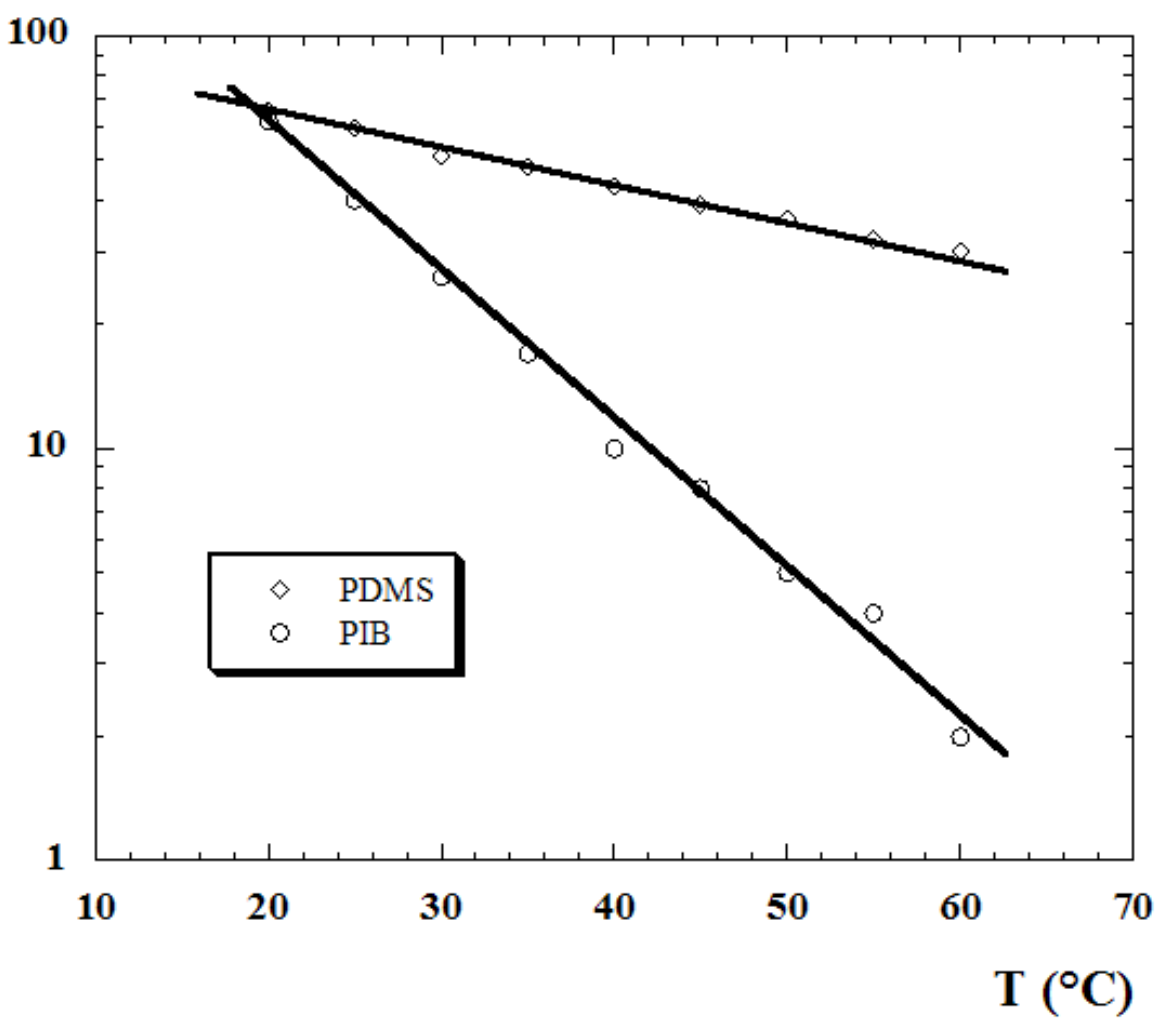

FIGURE 1. Polymer viscosities (PDMS and PIB) versus temperature. 
Since large changes are obtained, a semi-log scale was used which shows a typical linear dependence of the $\log$ (viscosity) as a function of temperature. As expected for PDMS, the viscosity change is not so large, going from $66 \mathrm{~Pa}$.s to $30 \mathrm{~Pa} . \mathrm{s}$ in the range $\left[20^{\circ} \mathrm{C}-60^{\circ} \mathrm{C}\right]$. On the other hand, the PIB viscosity shows larger changes in the same range, going from $62 \mathrm{~Pa}$.s (at $\left.20^{\circ} \mathrm{C}\right)$ to $2 \mathrm{~Pa} . \mathrm{s}\left(60^{\circ} \mathrm{C}\right)$ roughly. This interesting behavior allows to cover a viscosity ratio $\mathrm{p}$ going from about 1 to 15, when PDMS is the dispersed phase. On the other hand, when PDMS is the matrix, we cover the symmetric range from 0.067 to 1 . This allows us to recover the interesting range (Verdier, 2000), where the viscosity ratio $\mathrm{p}$ goes from about 0.1 to 10 . This will provide us another verification of the previous results, by changing temperature, rather than changing fluids. The interfacial tension between PDMS and PIB was obtained on a usual pendant drop apparatus (Digidrop), using PDMS inside PIB, with a temperature ranging between $20^{\circ} \mathrm{C}$ and $60^{\circ} \mathrm{C}$. Density measurements are required and were taken from previous experiments (Longin, 1999). The results for interfacial tension are presented in figure 2.

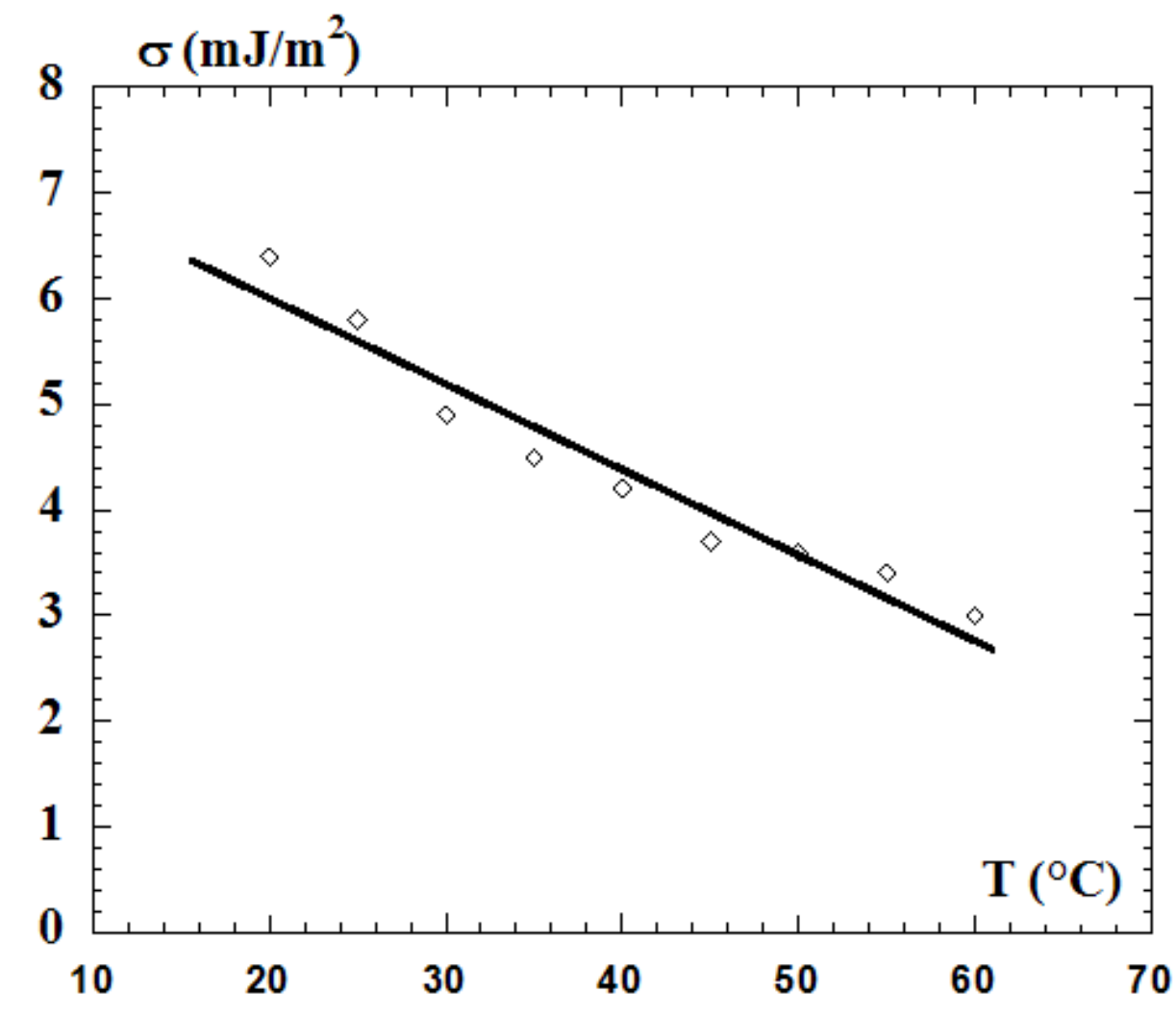

FIGURE 2. Interfacial tension as a function of temperature.

An approximately linear dependence is obtained as in the literature for usual polymer pairs (Wu, 1992). Data concerning the temperature dependence for this PDMS/PIB system has been published in the literature (Wagner and Wolf, 1993), and is in the range between 2.5 and $4 \mathrm{~mJ} / \mathrm{m}^{2}$ for PDMS/PIB systems, but it is strongly influenced by the molecular weight. It may also be a nonlinear function of temperature, as it passes through a maximum for some systems. On the other hand, it is close to $3 \mathrm{~mJ} / \mathrm{m}^{2}$ at room temperature for similar systems (Grizzuti, 1997). Our values are found slightly higher but in reasonable agreement to the first data in the same temperature range. This may be due to the different fluid range, or to the higher molecular weights for our fluids. Indeed the surface tension of each component usually increases with molecular weight.

\section{Droplet coalescence}


This technique has been previously investigated (Verdier, 2000) and was later improved (Verdier, 2001) to study the effect of the viscosity ratio $p=\eta_{1} / \eta_{2}$, where 1 refers to the droplet and 2 corresponds to the suspending medium. Six decades in viscosity ratio were covered and the collision time was introduced. Droplets are mixed slowly until a monodisperse emulsion is obtained. Then the system is let to relax until droplets are spherical, and also to let air bubbles out if present. Putting the system between a glass plate and a micro cover-glass allows proper visualization of the system (magnification x20 or x50 gives good quality pictures). There should be enough droplets but not too many, to avoid interactions between them. One can localize the droplets away from edges, so that end effects are avoided. Let $\mathrm{D}(\mathrm{t})$ be the length of the resulting axisymmetric droplet. A typical curve showing $D(t) / D_{0}$ is shown in Figure 3, as well as a sketch of the droplets.

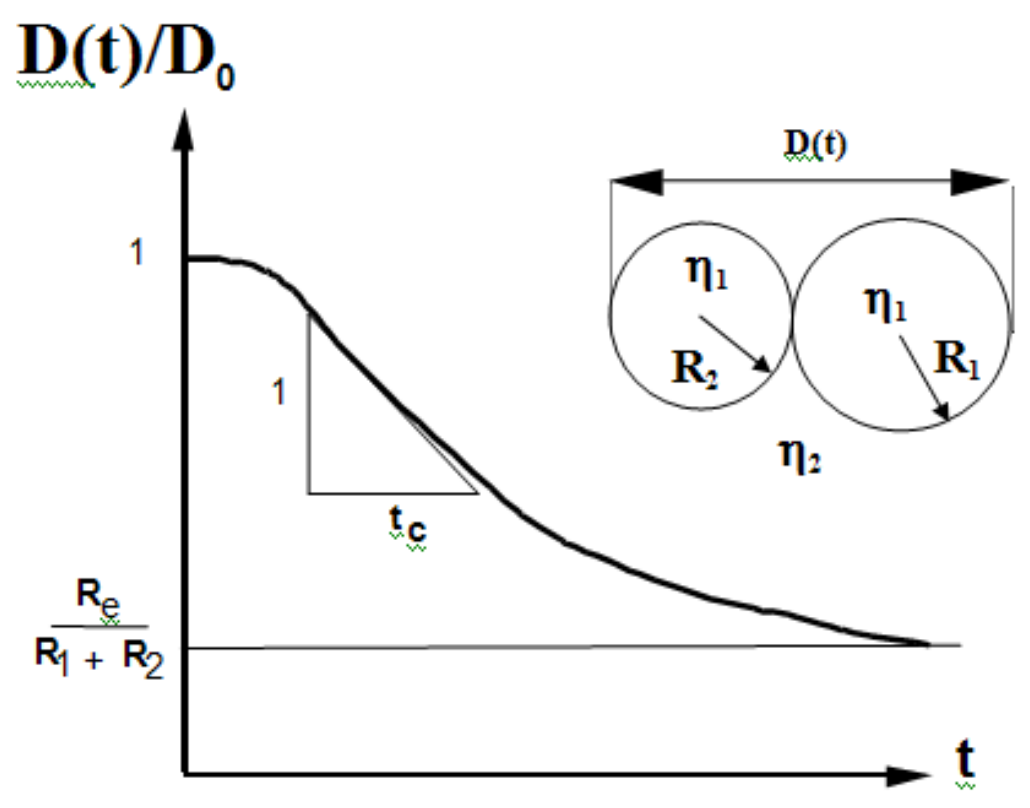

FIGURE 3. Sketch of the $\mathrm{D}(\mathrm{t}) / \mathrm{D}_{0}$ curve $\left(\mathrm{D}_{0}=2 \mathrm{R}_{1}+2 \mathrm{R}_{2}\right)$ and characteristic dimensions.

$\mathrm{D}_{0}$ is the initial length of this so-called composite droplet and equals $2\left(\mathrm{R}_{1}+\mathrm{R}_{2}\right)$, where $\mathrm{R}_{1}$ and $\mathrm{R}_{2}$ are the initial droplet radii just before the droplets coalesce. As in previous works (Verdier, 2000, 2001), we introduce a typical collision time, as the inverse of the negative slope at the inflexion point on the curve $\mathrm{D}(\mathrm{t}) / \mathrm{D}_{0}$ versus time $\mathrm{t}$, in other words :

$$
t_{c}=-D_{0} / D^{\prime}\left(t_{i}\right)
$$

This technique has been justified by the fact that the onset of collision $(t=0)$ is difficult to catch experimentally and therefore the method using the slope is more accurate. The evolution of the time dependence of the distance $\mathrm{D}(\mathrm{t})$ is followed using an optical microscope (Leica, DML model, magnification $\times 20$ or $\times 50$ ) using transmitted light, and recorded through the use of a camera (3-CCD color, KY-F55B JVC model, 25 frames/s) onto a videorecorder (U-Matic, SP type, VO-9600P model). Due to the values of the refraction indexes, no particular treatments or methods are required for observation. $\mathrm{D}(\mathrm{t})$ shows first a rapid decrease, until it eventually levels off due to the final relaxation of the droplet. The final stage can also be used for measuring the interfacial tension (Luciani et al., 1997) using a previous theory (Rallison, 1980). This latter 
technique is very valuable and works at small droplet deformation but it may be difficult to create an ellipsoid axisymmetric droplet. In our case, axisymmetric droplets have always been obtained due to the initial conditions, i.e. two spherical droplets.

\section{Influence of the radius size}

In previous works (Verdier, 2000, 2001), the same droplet sizes were used but this was a drawback of the technique. We present here another way to process the data, allowing for different droplet sizes. Since the collision time $t_{c}$ (figure 3 ) is the slope at the inflexion point, $t_{c}$ should be the time required for going roughly from $D_{0}=2\left(R_{1}+R_{2}\right)$ to $D_{\infty}=2 R_{e}$, where $R_{e}=\left(R_{1}{ }^{3}+R_{2}{ }^{3}\right)^{1 / 3}$ is the final radius of the resulting droplet, given by mass conservation. Therefore a typical relevant length scale R should be:

$$
\mathrm{R}=\left(\mathrm{R}_{1}+\mathrm{R}_{2}\right)-\mathrm{R}_{\mathrm{e}}
$$

Then let us use the following adimensional parameters. $p=\eta_{1} / \eta_{2}$ is the viscosity ratio and the reduced time of collision $t_{c}$ will be defined as $\sigma t_{c} / \eta_{1} R$. This definition is slightly different from the previous one (Verdier, 2000) but it allows to account for different droplet sizes.

\section{Collision time vs. viscosity ratio}

Several experiments at temperatures $20,25,30,35,40,45,50,55$, and $60^{\circ} \mathrm{C}$ were carried out to study droplet collision. No particular droplet sizes were chosen. Droplets already closed to each other were selected to minimize the time it takes for draining the outer fluid. The reduced time of collision obeys a law similar to the previous ones (Verdier, 2000, 2001). This is described in figure 4 , where $\sigma t_{c} / \eta_{1} R$ is plotted as a function of the viscosity ratio $\eta_{1} / \eta_{2}$ over two decades.

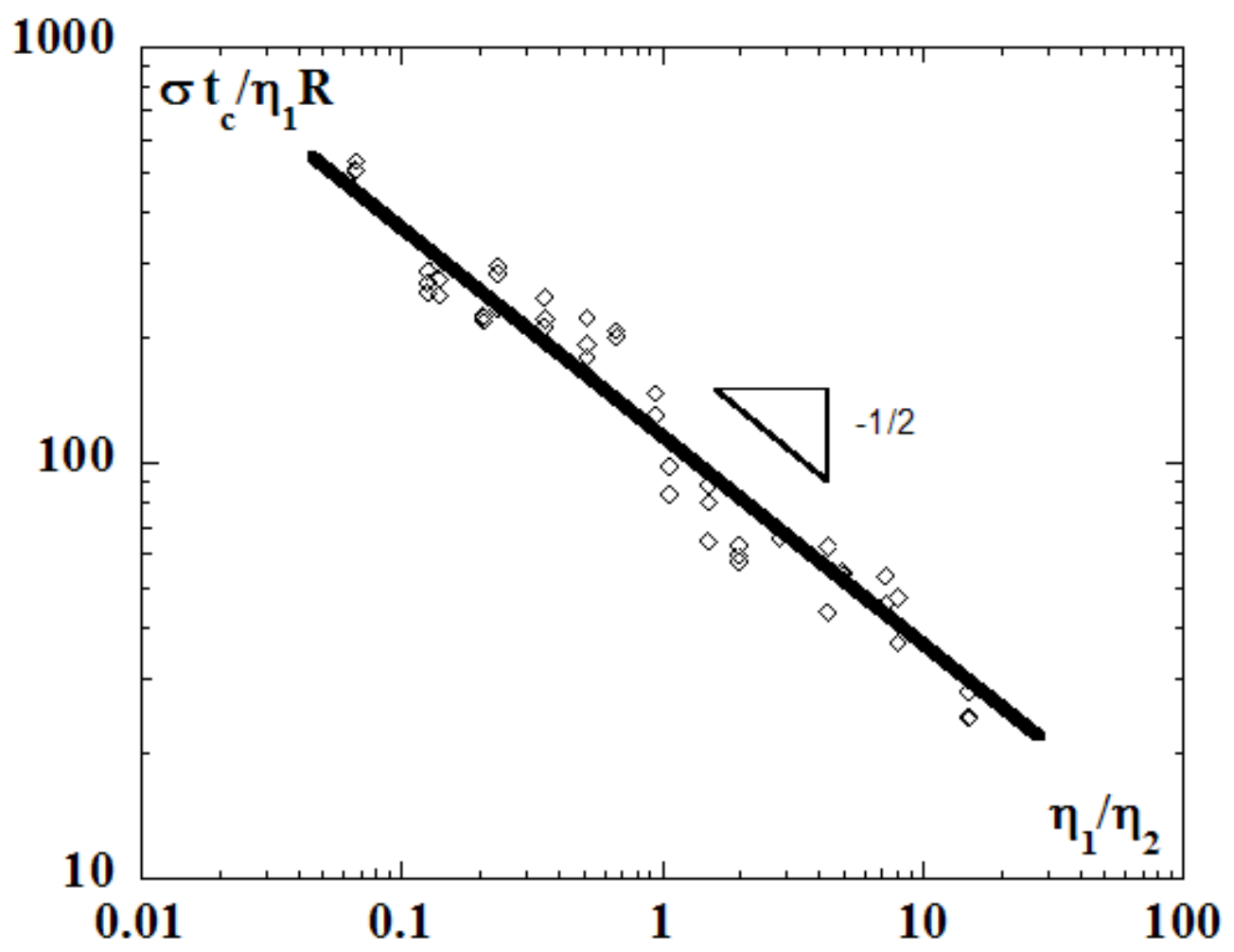

FIGURE 4. Nondimensional collision time vs. viscosity ratio $p=\eta_{1} / \eta_{2}$

The linear fit obtained shows a power law dependence of -0.5 , in agreement with the previous paper (Verdier, 2000), but the reduction technique is valid at all temperatures. The 
constant is somewhat different, because of the new length $\mathrm{R}$, chosen in the dimensional analysis. We find:

$$
\sigma t_{\mathrm{c}} / \eta_{1} \mathrm{R}=118.2\left(\eta_{1} / \eta_{2}\right)^{-0.5}
$$

All the data points seem to be aligned quite well and therefore it is possible to use this technique inversely to have access to the interfacial tension. Indeed, once the viscosities are known it is possible to invert (3) to have access to $\sigma$. On simply uses the following formula:

$$
\sigma=118.2 \mathrm{R} / \mathrm{t}_{\mathrm{c}} \sqrt{\eta_{1} \eta_{2}}
$$

The error on the measurement is rather small due to the fact that excellent accuracy is usually found both for times and radii measurements (through the microscope). This method is very promising for investigating the interfacial tension of polymeric materials, and the great advantage is that it does not require a sophisticated apparatus. Preparation is rendered easier, because of the simple mixing procedure, which does not require to insert droplets into another fluid using complicated devices.

Formula (4) is rather hard to predict theoretically, unless making assumptions. Nevertheless, using the idea that most of the flow close to the nip governs the viscous dissipations, one can assume lubrication in this region and show (Brochard and de Gennes, 1992, 2000) that the predicted reduced collision time varies like $\mathrm{p}^{-2 / 3}$ (Verdier, 2001). Unfortunately, this is not exactly the case here. Therefore, a more precise model requires the exact determination of the flow field, which is carried out next.

\section{PIV measurements}

\section{Fluid preparation and methodology}

Droplets were filled with small particles (CaCO3, size about 1 micron or smaller). Careful preparations were carried out, in order to have a homogeneoeus distribution of particles. We have limited our interest to the region inside the droplets, otherwise pictures would look too difficult to understand. Sometimes, it is possible that a few particles may appear outside droplets, but in a non significant manner. A limited number of particules is used so that no aggregation is possible. Also we want to make sure that the viscosities of the fluids do not change significantly. In order to achieve this mixing, particles are first isolated on a glass piece and spread out with a spatula so that they cannot aggregate. They are put in the oven and let to dry. After 2 hours, fluid 1 is mixed gently with the particles until a homogeneous mixture is obtained. Verification is done under the microscope. If it is satisfactory, then fluid 2 (i.e. the matrix) is added to fluid 1 , as in the case of the collision experiment described before. Again the system is put between the glass plate and micro cover-glass and ready for observation. The same procedure is used to achieve collision. Then films are used as an input to the PIV processing system (Dantec). Pictures are separated by $0.04 \mathrm{~s}$ at best and may be used for determining flow fields and streamlines. In order to have a good resolution, it is sometimes better to use more distant pictures (timewise) to obtain a sufficient particle displacement.

\section{Qualitative results}

An exemple of the flow field is shown in figures $5 \mathrm{a}$ to $5 \mathrm{f}$. The size of the droplets can be seen from the microscope scale. In addition, a second scale (arrow) has been added for the velocity magnitude. This is a non stationnary problem and streamlines need to be computed at each time step. In the series of pictures, the system was PDMS inside PIB at a temperature of $50^{\circ} \mathrm{C}$. 
Let us first point out that this case corresponds to droplets of equal sizes. Different droplet sizes will discussed later. In this case, the flow field is axially symmetric with respect to the (Or) axis, in cylindrical coordinates.

- Figure $5 \mathrm{a}$ is taken at time $\mathrm{t}=0.24 \mathrm{~s}$, very close to the start. One can see the very rapid motion of the neck, with velocities close to $4 \mu \mathrm{m} / \mathrm{s}$ already. The neck is showing a large curvature, as already mentioned in previous works (Frenkel, 1945, Kuczynski, 1949, Mazur and Plazek, 1994). Note also that motion within the droplets is starting slowly and that velocities are much smaller far from the neck.

- At a later time of $t=1 \mathrm{~s}$ (Figure $5 \mathrm{~b}$ ), the flow within the droplets has become more significant: the two droplets are merging. Velocities at the neck ring are very important, in the range of $20 \mu \mathrm{m} / \mathrm{s}$, probably close to their maximum value.

- The next picture is taken at $\mathrm{t}=1.48 \mathrm{~s}$ (Figure $5 \mathrm{c}$ ). The velocity at the neck is now decreasing slowly after having reached a maximum. The flow field near the centre of the droplets looks like a uniaxial elongational flow, with a time dependent stretching rate.

- Figure $5 \mathrm{~d}(\mathrm{t}=4 \mathrm{~s})$ shows the resulting droplet at the point where the neck just disappears. Again the flow is clearly of elongation type close to the center. Velocities are still decreasing.

- At $\mathrm{t}=5 \mathrm{~s}$ (Figure 5e), relaxation is starting. This experiment is comparable to the ones described previously (Luciani et al., 1997, Guido and Greco, 2001) and the droplet slowly starts to round like a sphere. Velocities are small everywhere (around $1 \mu \mathrm{m} / \mathrm{s}$ ).

- Time $\mathrm{t}=8 \mathrm{~s}$ shows an almost spherical droplet (Figure $5 \mathrm{f}$ ). The collision process is almost complete. Velocities are hardly measurable and are close to 0.

This gives an idea of the flow field inside droplets, but it is also of interest to show a complete picture of the flow field, inside and outside droplets. The overall flow field is needed in order to determine dissipations. 
a.

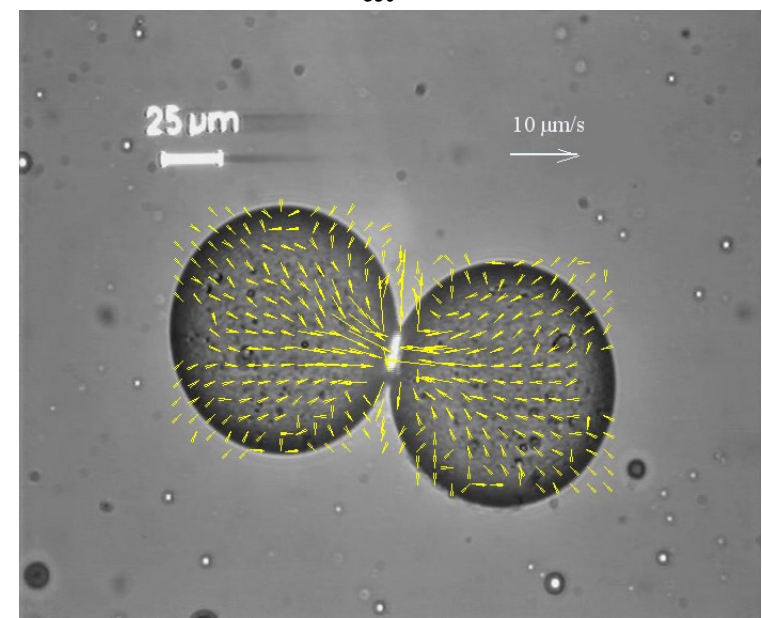

b.

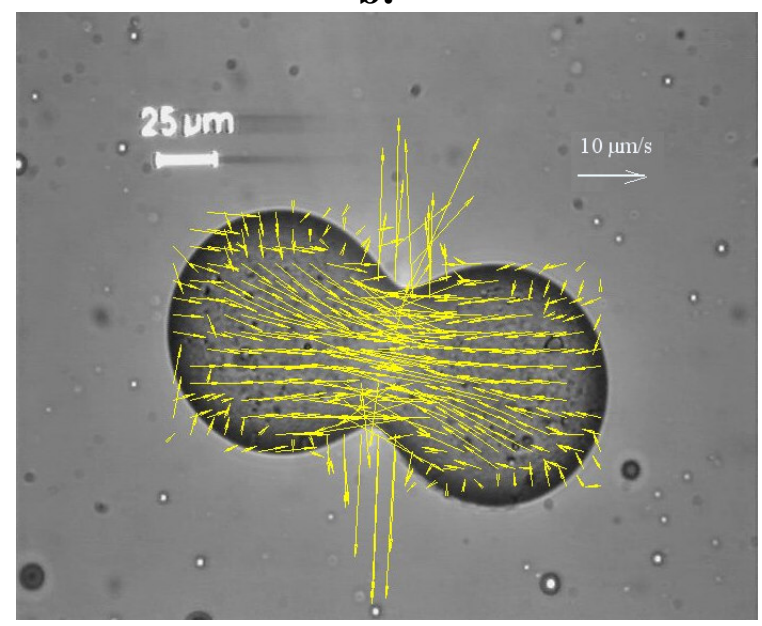

c.

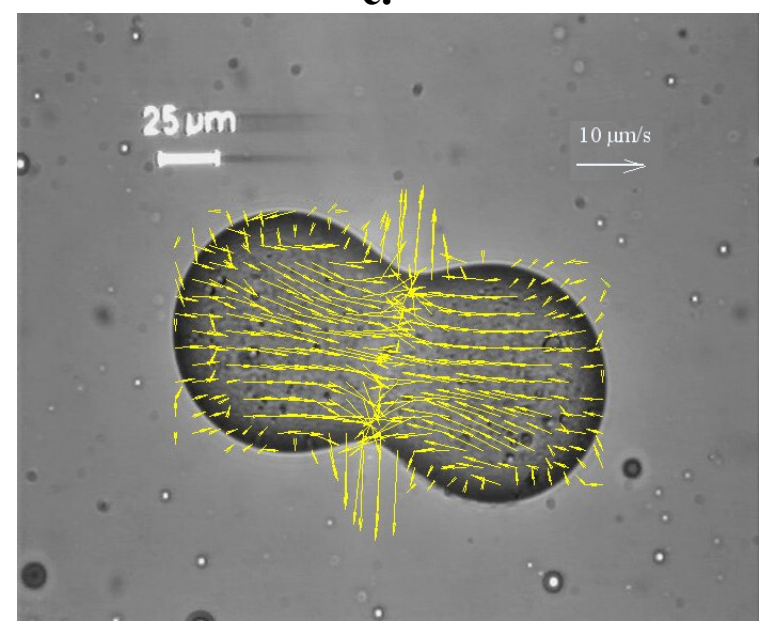

d.

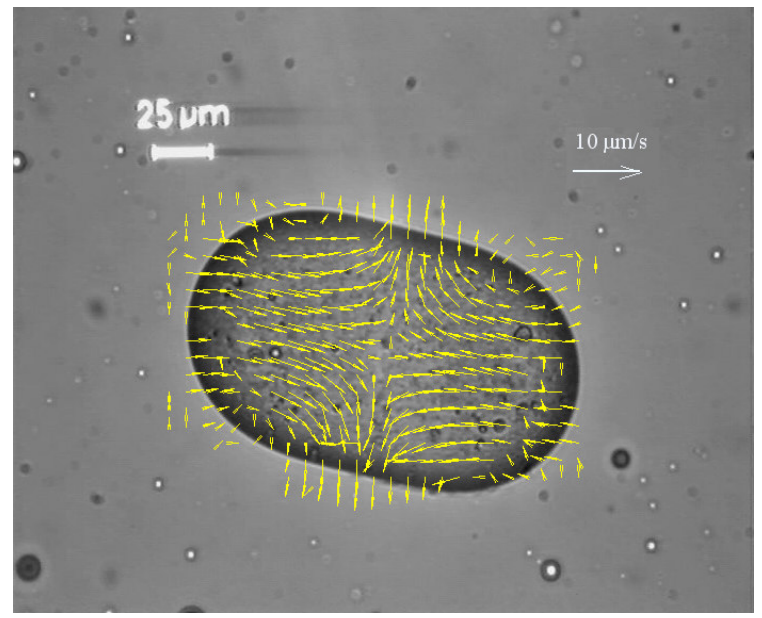

e.

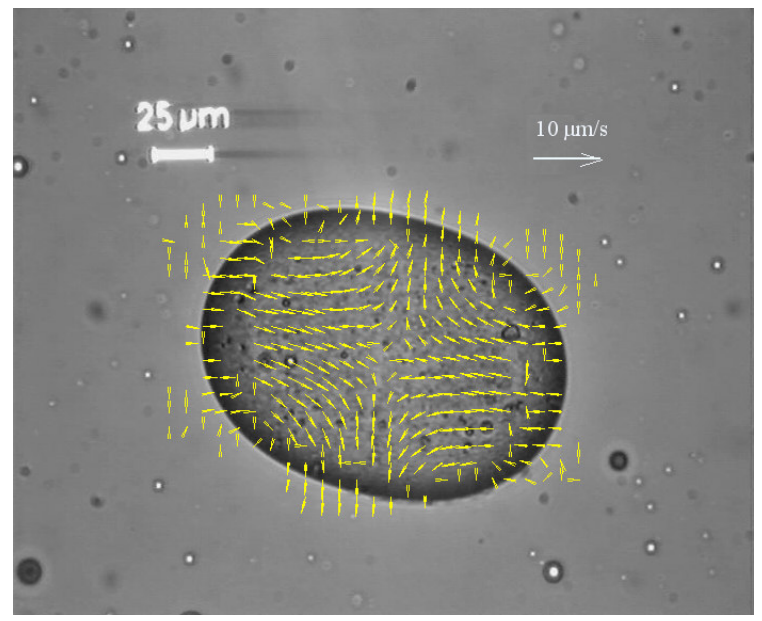

f.

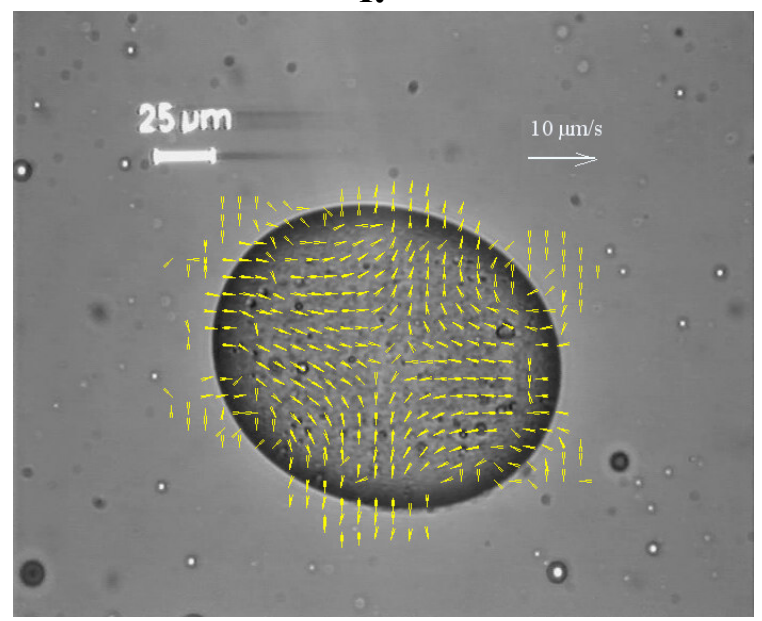

FIGURE 5. Flow field at time a. $t=0.24 \mathrm{~s}, \mathrm{~b} . \mathrm{t}=1 \mathrm{~s}, \mathrm{c} . \mathrm{t}=1.48 \mathrm{~s}, \mathrm{~d}$. $\mathrm{t}=4 \mathrm{~s}, \mathrm{e} . \mathrm{t}=6$., f. $\mathrm{t}=8 \mathrm{~s}$ (PDMS inside $\mathrm{PIB}, \mathrm{T}=50^{\circ} \mathrm{C}$ ). 
Figure 6 shows the overall velocity field. We have used particles inside the droplets and outside. Few are necessary otherwise the picture would become hard to read. Let us note the presence of the elongational flow region at the droplet centre, as discussed above, although it is not a uniaxial one, due to different droplet sizes. One can pay attention to the presence of recirculating flows outside the droplets, due to the fact that fluid 2 is displaced outwards at the neck, and on the other hand it is slowed down later in the surrounding fluid at rest. Fluid 2 comes back towards the droplet due to the interface motion which pulls it again towards the droplet centre. This creates a recirculating motion, at each given time $(\mathrm{t}=1.24 \mathrm{~s}$ here).

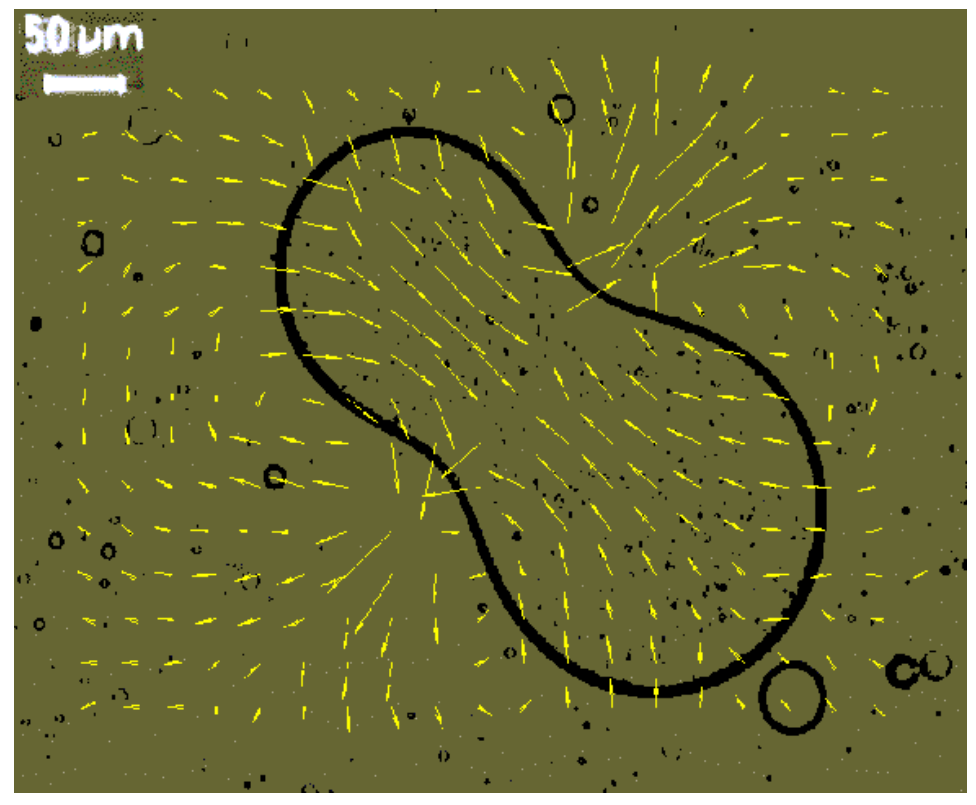

FIGURE 6. Complete velocity field (inside droplets and outside) for a PDMS droplet in PIB $\left(\mathrm{T}=50^{\circ} \mathrm{C}\right)$. Picture taken at $\mathrm{t}=1.24 \mathrm{~s}$.

\section{Velocity data}

The PIV technique presented is therefore a very good tool for monitoring the flow field within droplets. Also measurements at special points of interest can be made easily. Such points are the end points, the points on the neck ring, and the region at the center of symmetry which is also of interest. Data at such points is shown next.

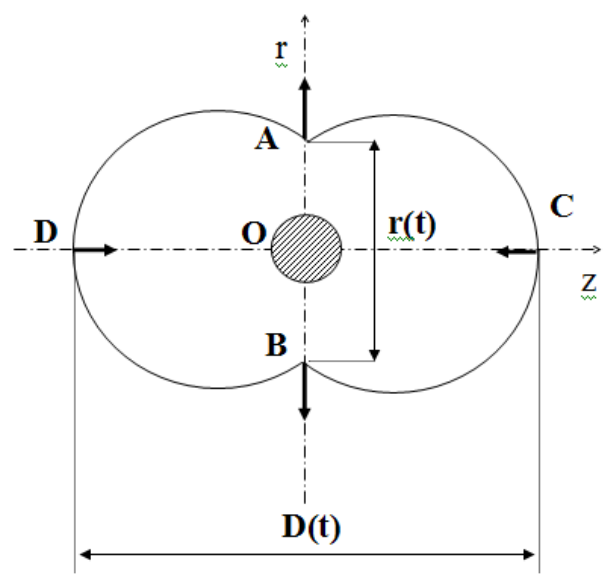

FIGURE 7. Characteristic picture of the composite droplet. $\mathrm{D}(\mathrm{t})$ and $\mathrm{r}(\mathrm{t})$ are also shown, as well as elongational flow region location (shadowed circle) and typical velocity vectors. 
At typical points defined in figure 7, the velocity vector is along the r-axis (A and B, points on the neck ring) and along the $\mathrm{z}$-axis ( $\mathrm{C}$ and $\mathrm{D}$, end points). The evolution of the velocities of these points, namely $\mathrm{V}_{\mathrm{A}}, \mathrm{V}_{\mathrm{B}}, \mathrm{V}_{\mathrm{C}}, \mathrm{V}_{\mathrm{D}}$, is shown in figure 8 , as well as the distance $\mathrm{D}(\mathrm{t})$ and the evolution of the distance corresponding to the neck $\mathrm{r}(\mathrm{t})$, defined in figure 7 . These velocities have been measured when possible, and in some cases, extrapolation techniques were used, because it is not always possible to have a particle velocity at the exact position. Nevertheless, this technique provides reasonable data, consistent with the photographs.

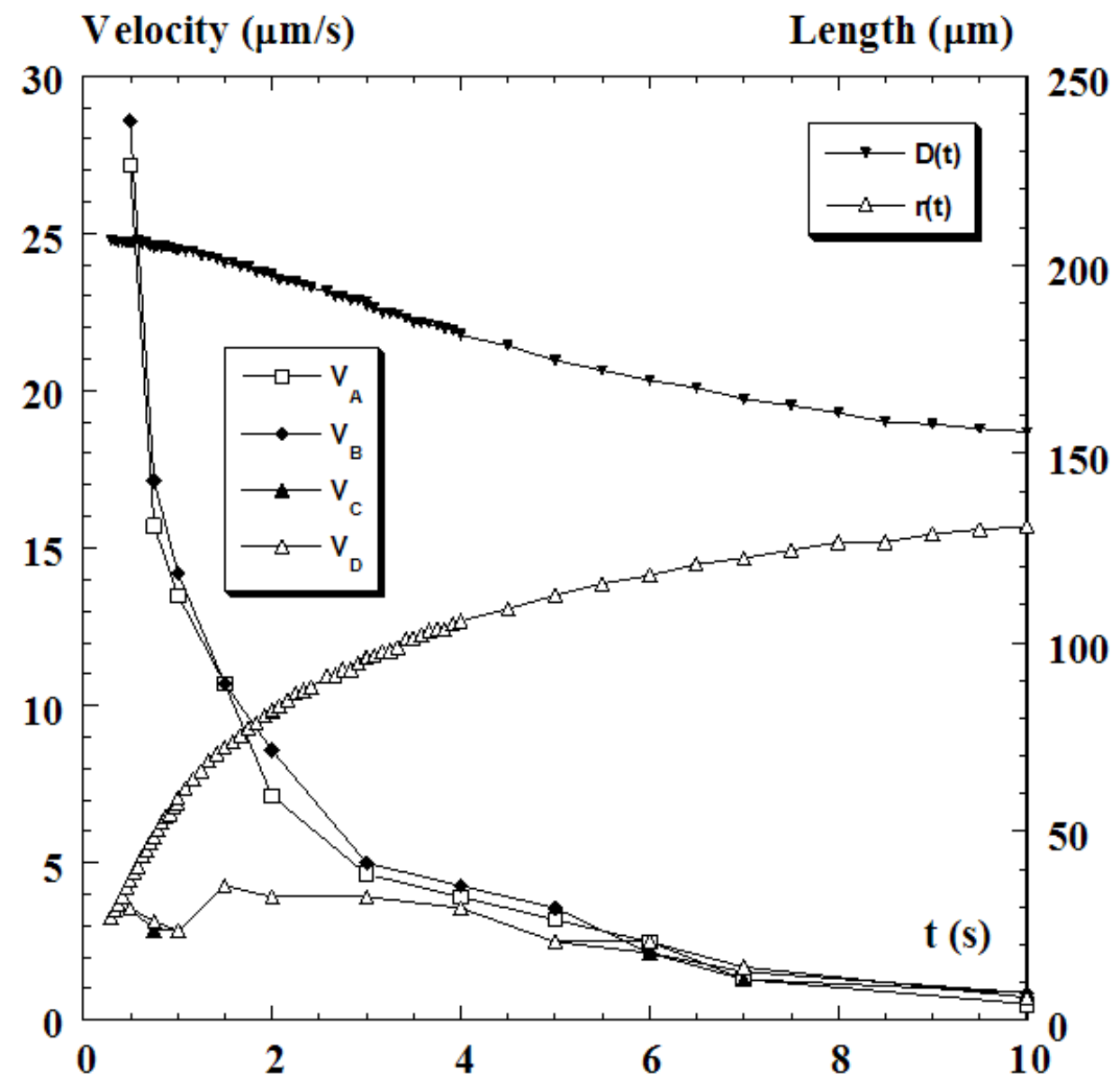

FIGURE 8. PDMS droplets in PIB $\left(\mathrm{T}=50^{\circ} \mathrm{C}\right)$. Characteristic velocities at points $\mathrm{A}, \mathrm{B}, \mathrm{C}, \mathrm{D}$. $\mathrm{D}(\mathrm{t})$ and $\mathrm{r}(\mathrm{t})$ also plotted vs. time $\mathrm{t}$.

The velocity of points A and B ("neck ring") is decreasing until it reaches zero. Still it should be pointed out that the velocity at such points is zero at the start, and increases very rapidly and should probably go through a maximum. Due to the uncertainly of measurements at small times, only confident data points are shown (above $0.5 \mathrm{~s}$ ).

At points $\mathrm{C}$ and $\mathrm{D}$, the velocity is most probably increasing first (before 1s) then shows a plateau (from $1 \mathrm{~s}$ to $4 \mathrm{~s}$ ) during the collision (or fusion) process, then finally drops out until it reaches zero. The final decrease is associated to relaxation of the droplet.

The distance $\mathrm{r}(\mathrm{t})$ has been used on several occasions and is also of great interest, as shown for example in the sintering problem, both theoretically and experimentally. Kuczynski (1945) and Frenkel (1949) showed that for a newtonian droplet in air, the distance varies like $t^{0.5}$ and is in agreement with experiments. On the other hand, when viscoelastic effects prevail (Mazur and Plazek, 1994), and if $\mathrm{r}(\mathrm{t}) \approx \mathrm{t}^{\mathrm{n}}$, then $\mathrm{n}$ can vary between 0.2 and 0.9 , and is related to the 
viscoelastic behaviour through the compliance. Finally Eggers (1998) found a theoretical exponent of $1 / 7$ and a numerical one of $1 / 6$. In our experiment, we determine the distance $r(t)$ which is plotted in figure 9 in log-log scale, in order to measure the exponent $\mathrm{n}$.

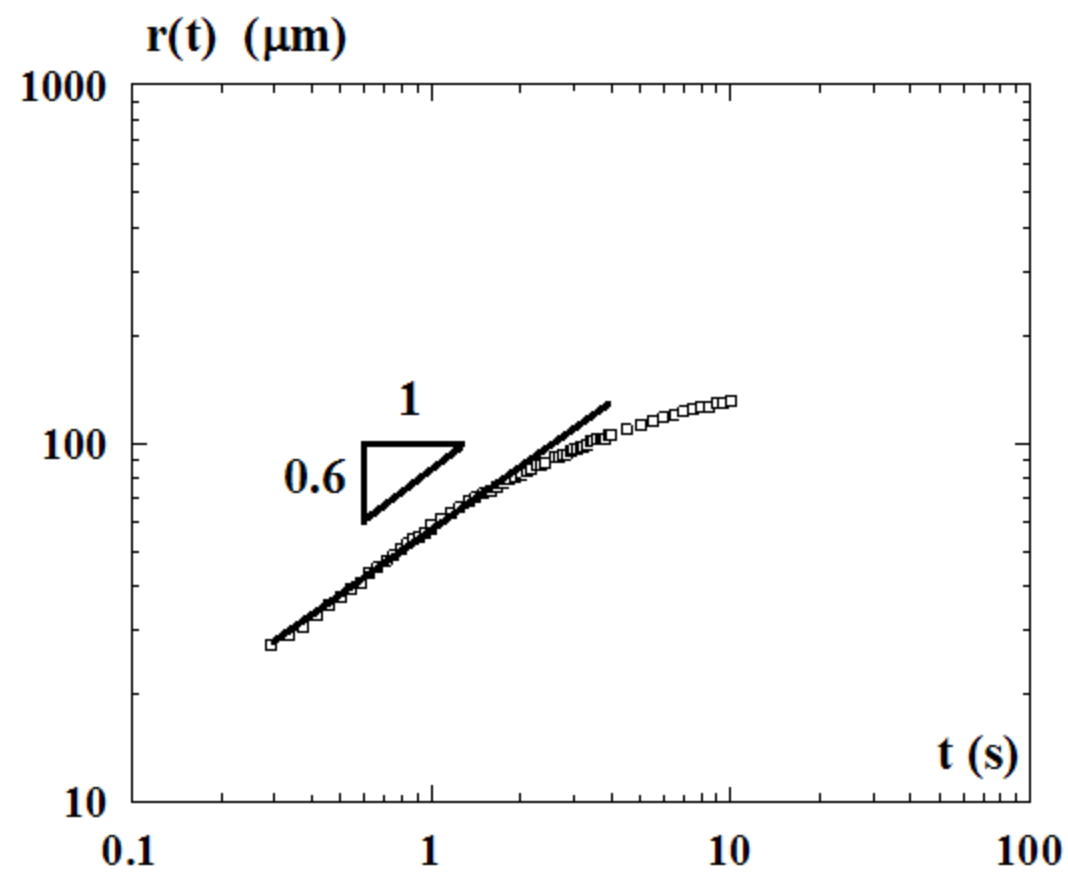

FIGURE 9. Distance $r(t)$ as a function of time $t$.

We have limited ourselves to data for which $r(t)$ is measurable, i.e. times larger that about $0.24 \mathrm{~s}$. In addition, there is light diffusion at the interface (Verdier, 2000) which is also observed for example in figure 5a, and this renders the measurements difficult. We find a power dependence (Figure 9) of the type:

$$
\mathrm{r}(\mathrm{t}) \approx \mathrm{t}^{0.6}
$$

This dependence is valid at small times and is in reasonable agreement with previous observations mentioned above, such as the ones corresponding to newtonian fluids. At larger times, the dependence is no longer valid but it is to be remembered that the theory (Frenkel, 1945) is only valid for short times, due to the assumptions on the initial droplet shapes. Also this exponent may depend on $\mathrm{p}$, which was not discussed in previous works.

Finally, data showing the velocity of points along the z-axis is shown in Figure 10. This is done at different times of $0.24 \mathrm{~s}, 1 \mathrm{~s}, 3 \mathrm{~s}$ and $8 \mathrm{~s}$. This data is relevant for discussing elongational flows.

The symmetry of the droplet with respect to the $(\operatorname{Or} \theta)$ plane (where $\theta$ is the usual polar angle) is emphasized again through the velocity profile symmetry. In addition, the central region of flow gives evidence of a linear dependence of the velocity versus distance $z$, as shown by the straight lines going through the data points. This is typical of an elongational flow, which has a velocity component proportional to the distance. In this graph, the width $\mathrm{L}_{t}$ of the linear region is also presented, which shows the size of the elongational region of flow. This will discussed in the next part. 


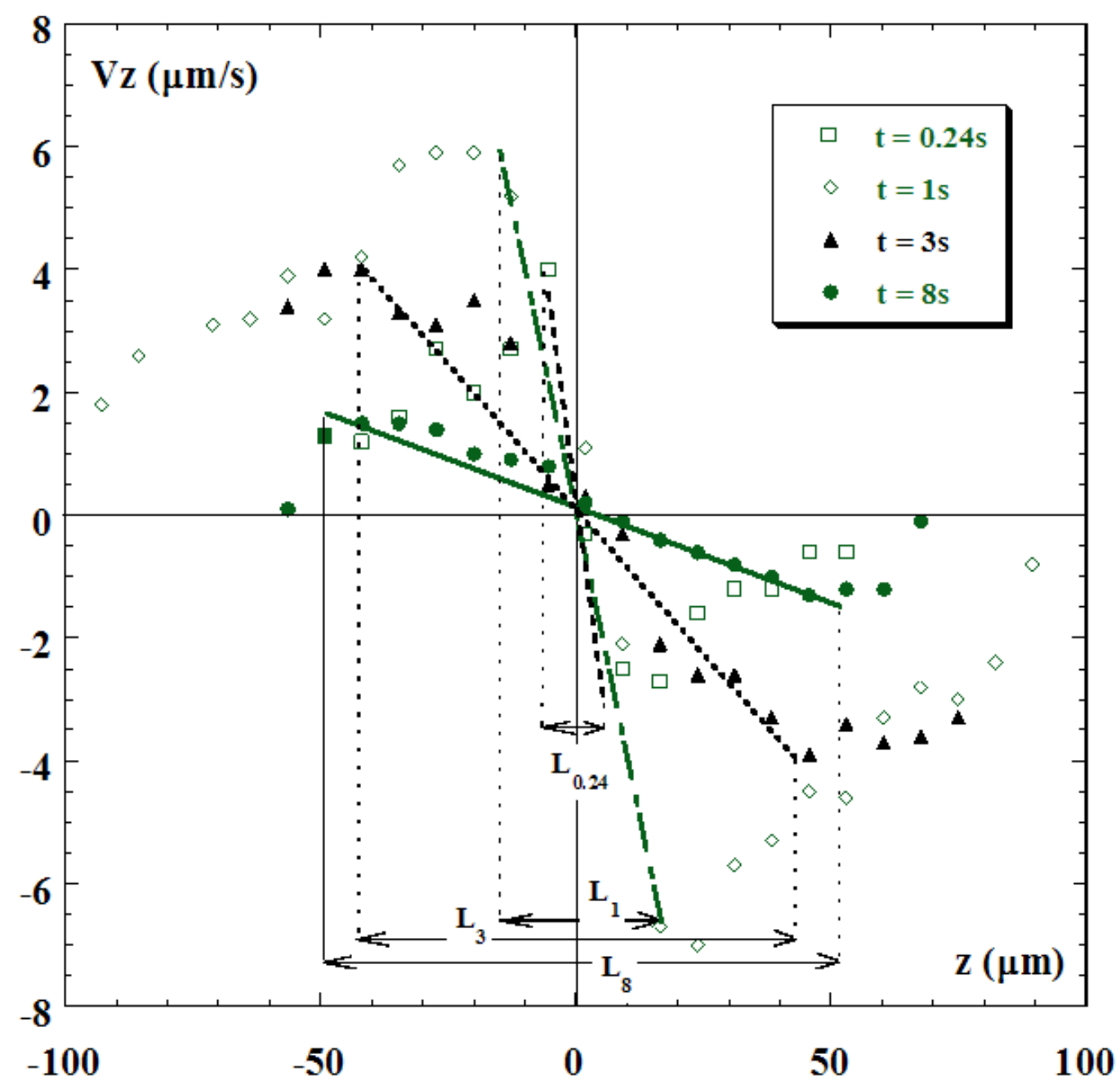

FIGURE 10. Velocity $\mathrm{V}_{\mathrm{z}}$ of the points along the z-axis. Straight lines are drawn to fit the data close to the centre, corresponding to the elongational region of length $\mathrm{L}_{\mathrm{t}}$ (data at times $\mathrm{t}=0.24 \mathrm{~s}, 1 \mathrm{~s}, 3 \mathrm{~s} 8 \mathrm{~s}$ ).

\section{Discussion}

The coalescence experiment discussed in this work has two interesting features associated with it. It is first shown to be a nice way to have access to a relationship between the collision time and the viscosity ratio through a simple relation. Therefore, it can used conversely, to determine the interfacial tension once the viscosities of the fluids are known. The only data needed is the time dependence of the resulting droplet length, from which the so-called collision time is determined. Previous methods for determining the interfacial tension between polymer melts can then be compared.

The spinning drop tensiometry (Elmendorp et de Vos, 1986, Joseph et al., 1992), the pendant drop method (see for example Kamal et Demarquette, 1997), the filament thread method (Cohen and Carriere, 1989), the embedded disk retraction method (Rundqvist et al., 1996) or ellipsoidal drop retraction (Luciani et al., 1997) all require a very well designed drop or filament to be inserted into another polymer, which is sometimes very difficult. In our instrument, all that is needed is to mix two polymers, without having to worry about shaping droplets. The drainage part (Chesters, 1991) is of course the longest part, but since many droplets can be chosen, this problem is properly handled. Finally, the collision process may require a long time, but it is to be remembered that 
only the first (short) part is of interest, until the inflexion point on the $\mathrm{D}(\mathrm{t})$-curve is found. Note also that some methods (i.e. spinning droplet, pendant drop) also require very long times when used with highly viscous polymers. Therefore this technique, coupled with a high temperature control now available with microscopes, is a very promising one for measuring the interfacial tension, as well as the relaxation properties.

It may also provide valuable information such as the diffusion of copolymers when inserted at the interface or elsewhere in the fluid. Indeed, the time dependence of the drop size change has been shown to be a valuable tool for measuring diffusion coefficients of copolymers using a spinning drop tensiometer (Gaines and Bender, 1972, Verdier et al., 2000).

The second important part of the experiment has been devoted to understanding flow fields using Particle Image Velocimetry (PIV). The methodology is shown to be well adapted for measuring flow fields in small-scale systems, as long as the particles are small enough (order of a micron or smaller if possible). Using droplets of diameters about $100 \mu \mathrm{m}$ seems to be a good compromise. The velocity field is time dependent, and reveals the presence of an elongational region in the centre of the droplets (region defined in figure 7). Due to the linear relationship obtained between $\mathrm{v}_{\mathrm{z}}$ and $\mathrm{z}$ (figure 10) at a given time, we postulate a flow field of the following type in the central region, when droplets are of equal size:

$$
\mathrm{v}_{\mathrm{r}}=\dot{\varepsilon} \mathrm{r} \quad \mathrm{v}_{\theta}=0 \quad \mathrm{v}_{\mathrm{z}}=-2 \dot{\varepsilon} \quad \mathrm{z}
$$

$\varepsilon$ is the elongational rate, a function of time, in the uniaxial elongational region and has been

chosen so that it is always positive in our case. $\varepsilon$ may be deduced from figure 10 using formula (6). The fact that the azimuthal component $v_{\theta}$ is zero is related to our observations but also seems natural. All the films made have demonstrated that no apparent distorsion or droplet shear with respect to the other axes exist: the only shear is in the (Orz) plane. At least this is what is observed during the experiment time, and is certainly true close to the central region. The component along the $\mathrm{z}$-axis is chosen to be proportional to $\mathrm{z}$, as shown in figure 10, and the r-component of the velocity is simply chosen to satisfy a uniaxial elongational flow, as well as mass conservation.

The size of the elongational flow region is time dependent, as shown in figures $5 \mathrm{a}-5 \mathrm{f}$. By simple inspection of the flow field, it can be deduced that the region of elongation is very small at the beginning and that it is larger and larger as time goes on. Indeed, figures $5 \mathrm{~d}-5 \mathrm{f}$ show that the elongational flow region has almost completely invaded the droplet volume. This is again verified in figure 10, where the size $L_{t}$ of the region of elongation at time $t\left(v_{z}\right.$ is a linear function of $\left.z\right)$ is shown for $\mathrm{t}=0.24 \mathrm{~s}, \mathrm{t}=1 \mathrm{~s}, \mathrm{t}=3 \mathrm{~s}, \mathrm{t}=8 \mathrm{~s}$. This size is increasing with time. Qualitatively, this region is close to a spherical domain (from figures 5a-5f). Finding an elongational region has been also discussed previously (Rallison, 1980). Note that for different droplet sizes, this flow field does not obey the same formula (6), but may probably be deduced by rescaling $\mathrm{r}$ and $\mathrm{z}$. Figure 6 shows that the elongational region still exists, but is no longer symmetric.

The dependence of the flow field on time (for equal droplets) can be postulated to be of the type $\dot{\varepsilon}=\dot{\varepsilon}(\mathrm{t})$, still in the neighborhood of point $\mathrm{O}$ (Figure 7). It is hard to give a simple form of $\dot{\varepsilon}(\mathrm{t})$, as only few experiments can be detailed here, but it is reasonable to think that the magnitude of the

flow is related to $\varepsilon(\mathrm{t})$, and that $\varepsilon(\mathrm{t})$ is a rapid increasing function of time then a decreasing one (figure 11), after reaching a maximum in between. This is also checked by looking at velocity of point A. Figure 11 shows the expected variation of $\varepsilon(\mathrm{t})$, with a few data points taken from the linear slope of $\mathrm{v}_{\mathrm{z}}$ in figure 10 . 


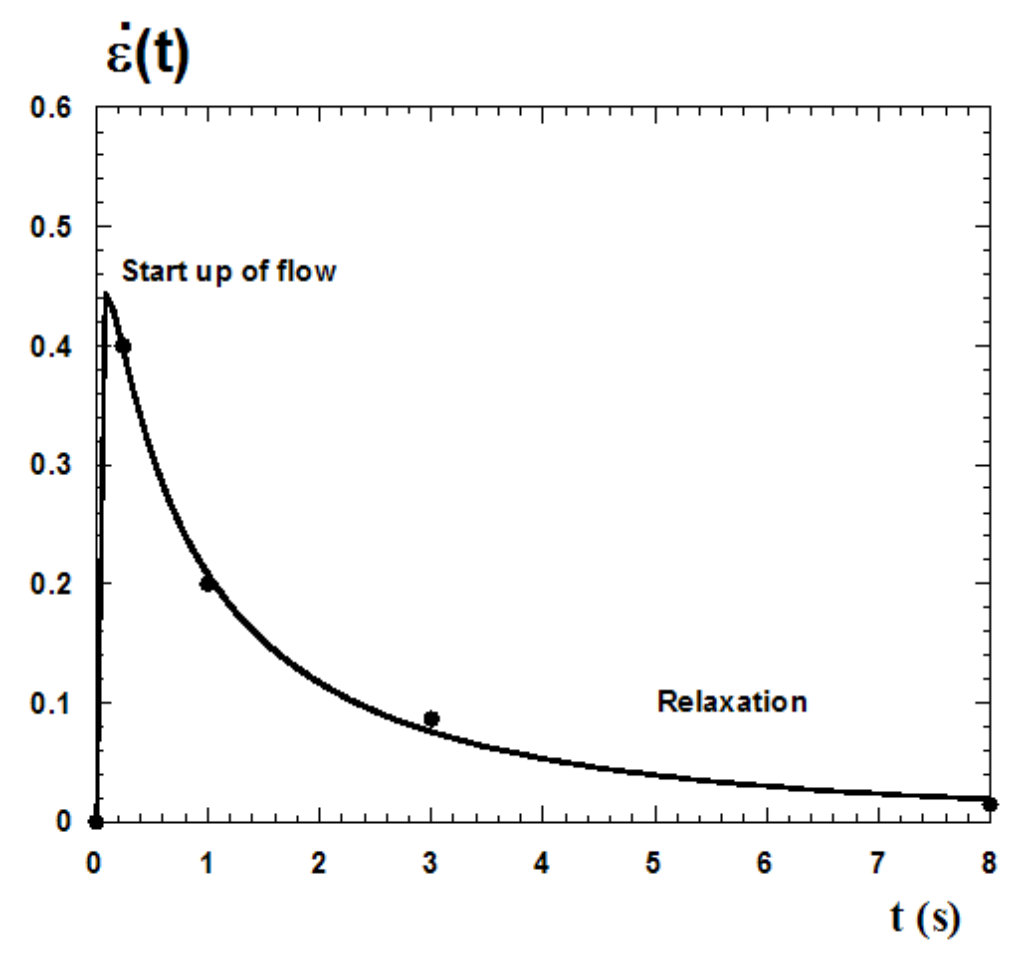

FIGURE 11. Typical dependence of $\dot{\varepsilon}(\mathrm{t})$ as a function of time (points calculated from figure 10). The line is only a guide for the eye.

Finally, the measurement of $\varepsilon(\mathrm{t})$ may be very useful to have access to other rheological properties. In particular, at larger times, figure 11 may be used to determine the time-dependent properties of a two-fluid system, but this requires more attention and is not the purpose of the present study. Such analyses have been attempted before (Joseph et al., 1992) and reveal the complexity of such two-phase systems. The interface viscoelasticity may also be investigated. But the main idea would be to consider other viscoelastic systems for such purposes.

Another final comment to be made is the fact that this accurate determination of the flow field should allow to calculate the dissipations at the neck, and this may be useful to model the dependence of the collision time as a function of the viscosity ratio theoretically.

\section{Conclusions}

In this study it has been demonstrated that the coalescence experiment is a very useful tool for determining the interfacial tension of a polymer-polymer system. The effect of the viscosity ratio and droplet sizes has been understood. In addition, the system has been investigated further to evaluate velocity fields. It is clearly shown that the flow field within droplets is a timedependent one, close to a uniaxial extension inside the droplets.

These results may be used further to determine the interfacial tension of more complex systems (with copolymers, different architectures, chain lengths, etc.). Results giving the flow field may also be useful for comparison with numerical simulations involving droplet deformations.

\section{Acknowledgments}

The authors are very thankful to Hélène Galliard, for helpful contribution to the PIV experiments. 


\section{References}

Brochard-Wyart F, de Gennes PG (1992) Dynamics of partial wetting, Advances in Colloid and Interface Science 39:1-11

Chesters AK (1991) The modeling of coalescence processes in fluid-liquid dispersions: A review of current understanding. Trans I ChemE 69(A):259-270

Cohen A, Carriere CJ (1989) Analysis of a retraction mechanism for imbedded polymeric fibers. Rheol Acta 28:223-232

De Gennes PG, Brochard-Wyart F (2000) Unpublished result

Eggers J (1998) Coalescence of spheres by surface diffusion. Phys Rev Letters 80(12):2634-2637

Elmendorp JJ, de Vos G (1986) Measurement of interfacial tensions of molten polymer systems by means of the spinning drop method. Polym Eng Sci 26(6):415-417

Frenkel J (1945) Viscous flow of crystalline bodies under the action of surface tension. J Physics USSR 9(5): 385-391

Gaines GL, Bender GW (1972) Surface concentration of a styrene-dimethylsiloxane block copolymer in mixtures with polystyrene. Macromolecules 5(1):82-86

Grace HP (1982) Dispersion phenomena in high viscosity immiscible fluid systems and application of static mixers as dispersion devices in such systems. Chem Eng Commun 14:225-277

Guido S, Greco F (2001) Drop shape under slow steady shear flow and during relaxation. Experimental results and comparison with theory. Rheol Acta 40:176-184

Janssen JMH, Meijer HEH (1995) Dynamics of liquid-liquid mixing: A 2-zone model. Polym Engng Sci 35(22):1766-1780

Joseph DD, Arney MS, Gillberg G, Hu H, Hultman D, Verdier C, Vinagre TM (1992) A spinning drop tensioextensiometer. J Rheol 36(4):621-662

Kamal MR, Demarquette N, Lai-Fook RA, Price TA (1997) Evaluation of thermodynamic theories to predict interfacial tension between polystyrene and popropylene melts. Polym Eng Sci 37(5):813-825

Kuczynski GC (1949) Self-diffusion in sintering of metallic particles. Transactions AIME 185:169-178

Longin P-Y (1999) PhD Thesis, Université Grenoble I. "Caractérisation ultrasonore de polymères et d'alliages de polymères".

Luciani A, Champagne MF, Utracki LA, Interfacial tension coefficient from the retraction of ellipsoidal drops (1997) J Polym Sci B: Polym Phys 35:1393-1403

Mazur S, Plazek DJ (1994) Viscoelastic effects in the coalescence of polymer particles. Prog Organic Coat 24:225-236

Rallison JM (1980) Note on the time-dependent deformation of a viscous drop which is almost spherical. J Fluid Mech 98:625-633

Rundqvist T, Cohen A, Klason C (1996) The imbedded disk retraction method for measurement of interfacial tension between polymer melts. Rheol Acta 35:458-469

Taylor GI (1934) The formation of emulsions in definable fields of flow. Proc Royal Soc 29:501-523

Verdier C (2000) Coalescence of polymer droplets: experiments on collision, C R Acad Sci IV 1:119-126

Verdier C (2001) The influence of the viscosity ratio on polymer droplet collision in quiescent blends. Polymer 42:6999-7007

Verdier C, Vinagre HT, Piau M, Joseph DD (2000) High temperature interfacial measurements of PA6/PP interfaces compatibilized with copolymers using a spinning drop tensiometer. Polymer 41:6683-6689

Vinckier I, Moldenaers P, Terracciano AM, Grizzuti N (1998) Droplet size evolution during coalescence in semi-concentrated model blends. AIChE J 44(4):951-958

Wagner M, Wolf BA (1993) Interfacial tension between Poly(isobutylene) and Poly(dimethylsiloxane) : Influence of chain length, temperature, and solvents. Macromolecules 26(24):6498-6502

Wu S (1982) "Polymer Interface and adhesion". New-York, Marcel Dekker Ed. 